\title{
Interaction of various functional elements in thin-walled cups formed by a sheet-bulk metal forming process
}

\author{
Robert Schulte ${ }^{1, a}$, Thomas Schneider ${ }^{1}$, Michael Lechner ${ }^{1}$ and Marion Merklein ${ }^{1}$ \\ ${ }^{1}$ Institute of Manufacturing Technology (LFT, Friedrich-Alexander Universität Erlangen-Nürnberg), Egerlandstrasse 13, 91058 Erlangen, \\ Germany
}

\begin{abstract}
Sheet-bulk metal forming allows the manufacturing of sheet metal parts with integrated functional elements. The investigated process combines deep drawing and upsetting. The occurring 2D and 3D stress and strain states lead to challenges regarding material flow control due to different geometries of functional elements. The numerical analysis shows the transferability for the results of the different functional elements for the ir combination and the interaction between those functional elements regarding the material flow. The results are validated by experimental tests.
\end{abstract}

\section{Introduction}

Legal frameworks and social change request lowemission products [1]. Especially the automotive industry is confronted with great challenges with regard to the application of resources both in the production and in the usage of modern vehicles [2]. For that reason, lightweight construction is increasingly important to enhance material efficiency to reduce fuel consumption [3]. However, a consistent implementation of lightweight construction is accompanied by increased functional integration of the parts manufactured especially in the powertrain [4]. This requires a higher load capacity. Therefore, the application of steel as workpiece material is favorable although current metal forming processes meet their limits with regard to cost and time expenditure due to insufficient process understanding [5]. The combination of conventional sheet and bulk metal forming processes to the class of sheet-bulk metal forming processes is a promising approach to realize lightweight parts with enhanced functional integration. A lack of process knowledge requires the investigation of sheet-bulk metal forming operations and process combinations as these have proofed their potential to expand the limits of conventional forming processes [6]. To shorten process chains and enhance the robustness of these processes fundamental aspects have to be investigated [7].

The investigated sheet-bulk metal forming process is a combined deep drawing and upsetting process which enables the single-step manufacturing of cups with various functional elements on the outer surface. These parts take up technical requirements of powertrain parts, such as synchronizer rings. The cups are manufactured with an external gearing, open carriers and a combination of both. For the forming of both types of functional elements the material flow control is the main challenge. The external gearing is a thick-walled element which requires a large thickening of material in the gear cavity [8] whereas for the open carrier, as a thin-walled element, excessive thinning of the material has to be prevented [9]. The combination of different functional elements now takes account of the increasing demand of functional integration and is presented in figure 1.

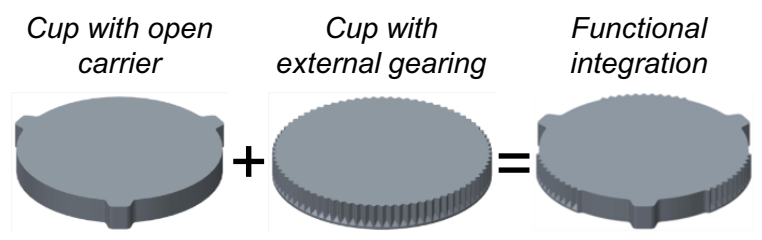

Figure 1: Combination of various functional elements in an integrated part geometry

Prerequisite for a successful manufacturing of the parts is the understanding the interactions of various functional elements regarding the material flow and defining of requirements for semi-finished parts. The layout is crucial for the deep drawing process as it has a strong influence on the draw-in behavior and also for the upsetting process as areas with different wall thickness have to be realized. Therefore, the material flow is investigated by numerical simulations and subsequently validated with experimental results. Relevant process limits for the forming of parts with an increased functional integration are shown and challenges are derived. An adaption of the blank layout is executed to enhance the material flow control and to meet the challenge.

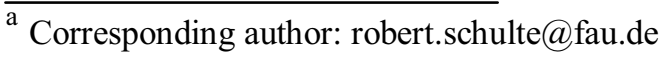




\section{Component characteristics forming concept \\ and}

This section gives an overview of part geometry, forming process, modelling and methodology to provide the necessary information for the investigations performed and discussed.

\subsection{Part geometry}

Figure 2 shows the investigated demo-part, which is designed according to a rising demand for parts with enhanced functional integration. The part investigated has three open carriers, arranged in an angle of $120^{\circ}$. Between those carriers the gearing is located. The number of teeth in a single section is 15 . The various functional elements are arranged cyclically and symmetrically. The inner diameter of the cup with the external gearing amounts to $d_{d p}=75.5 \mathrm{~mm}$ whereas the open carrier has an inner diameter of $d_{c}=85 \mathrm{~mm}$. The radius of the drawing punch is $r_{d p}=1 \mathrm{~mm}$. For the cup wall and the open carrier the maximum thickness amounts to $t_{\mathrm{wmax}}=2.25 \mathrm{~mm}$ which corresponds to the drawing gap, whereas the maximum thickness in the tooth tip is $t_{\text {tmax }}=3.75 \mathrm{~mm}$. The outer transition radius from the functional elements to the round cup is $\mathrm{R} 2=2 \mathrm{~mm}$, the inner radius is $\mathrm{R} 1=1 \mathrm{~mm}$. The height of the cups depends on the geometry of the semi-finished product and on the forming force applied.

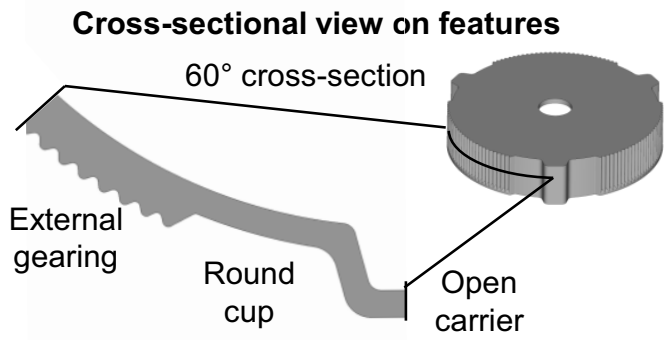

a) Gearing

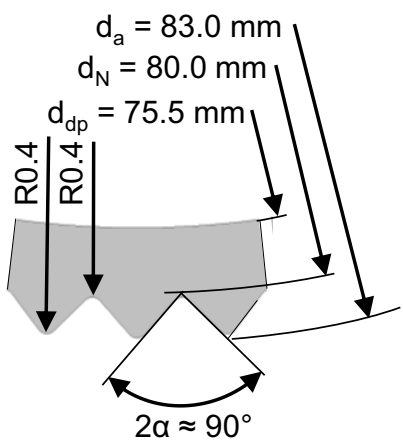

b) Open carrier

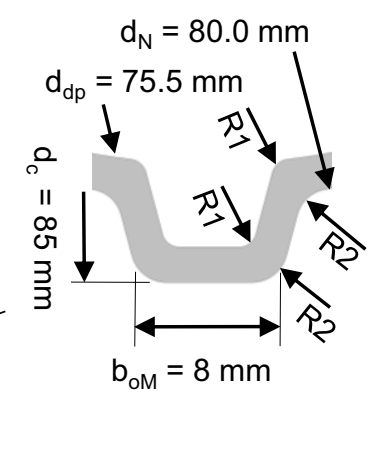

Figure 2: Modelling of drawing die for open carrier (a) and gearing (b)

\subsection{Process set-up}

To manufacture parts with an increased functional integration the tool concepts presented in [8] for the external gearing and [9] for open carriers are combined. The tool concept consists of an upper tool with drawing die and upsetting punch and a lower tool with upsetting plate and drawing punch. In addition, a hydraulic cylinder is connected to the upsetting punch. Within the manufacturing process the semi-finished product is positioned on the center of the drawing punch and then clamped by the upsetting punch in axial direction to avoid a lifting of the bottom of the cup from the drawing punch caused by bending stresses induced into the part at the beginning of the upsetting operation. The clamping force $F_{c}$ is applied by the additional hydraulic cylinder. The tool setup is presented in figure 3 . The cup is formed as the upper die cushion of the triple acting hydraulic press is continuously positively displaced under the drawing force $F_{d}$ and the drawing die reaches a mechanical stop at the upsetting plate. Subsequently, the forming force is transmitted into the upsetting punch by a mechanical stop and the drawing punch is displaced by the upsetting force $F_{u}$. To perform the necessary kinematics a triple-acting hydraulic press Lasco TZP 400/3 is used. In the experimental set-up the deep drawing process proceeds stroke-controlled and the upsetting process proceeds force-controlled. The process set-up and tool components are shown in figure 3. a)

Initial
position

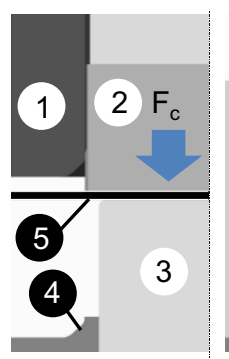

(1) Drawing die

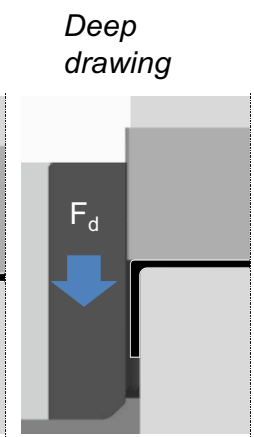

(2) Upsetting punch
(4) Upsetting plate

\section{(5) Blank}

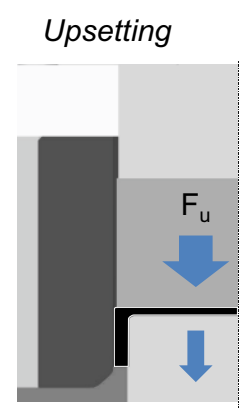

3) Drawing punch

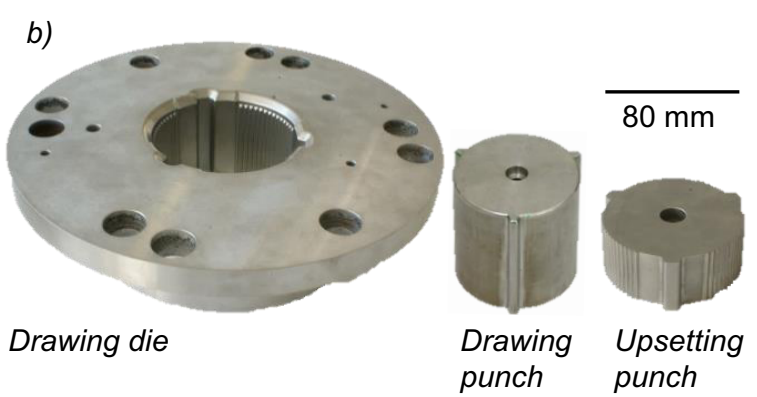

Figure 3: Cross-section of tool concept for deep drawing and upsetting (a) and tool components for experimental process setup (b)

Both, the cup and the thin-walled element open carrier are formed in the deep drawing operation, whereas the gearing as a thick walled element is formed in the upsetting process. The open carrier is formed as workpiece material is drawn over the drawing punch. During upsetting the sheet thickness in round cup and open carrier exceeds the initial sheet thickness slightly as the thin-walled functional elements are formed during deep drawing and calibrated in the upsetting process. The gearing is formed under compression stress during 
upsetting, as the cup height is reduced with ongoing stroke the material is forced to flow radially into the gear cavity. With an increased contact zone between tool and workpiece the material flow into the bottom of the cup and burr formation increase as friction and yield stress are rising.

\subsection{Modelling}

For a fundamental investigation of the material flow the process set-up is transferred to a numerical model. For the numerical investigation the implicit FE-code simufact.forming 12 is used. The workpiece material applied is DC04 and the initial sheet thickness is $\mathrm{t}_{0}=2 \mathrm{~mm}$. The flow curve is determined in a layer compression test and the flow criterion is modelled according to von Mises with isotropic hardening. The tools are modelled as rigid bodies. In the numerical simulation the forming process proceeds strokecontrolled, so that analyses at any force or stroke are possible.

Due to the cyclical and symmetrical arrangement of the functional elements a $60^{\circ}$-section of the workpiece geometry is used to model the process in the numerical simulation. The symmetry planes of the model are positioned in the middle of open carrier and gearing. To prevent contact problems at the interfaces and to enhance numerical stability a larger tool segment is used. The tool segment modelled is $90^{\circ}$ wide. The numerical model allows a tool set-up without gaps so burr formation can be eliminated. As the material flow into the die cavity is investigated the material flow into the bottom of the cup is not considered. The inner diameter of the blanks applied is set to $70 \mathrm{~mm}$ and the inner section is filled with a dummy plate which is modelled as rigid body. This adaption allows a decreased number of elements and hereby a decreased calculating time.

The contact condition between modified part and dummy plate is defined as glue contact. In contrast to the experimental set-up the numerical process is divided into the two process steps deep drawing and upsetting to leave out passive components and decrease the calculation time. Furthermore, this enables a specific adaption of the meshing for sheet- and bulk metal forming operations.

The final conditions of the deep drawing process are the initial conditions for the upsetting operation. Tool position, workpiece geometry, mesh, forming history and residual stresses are transferred. For deep drawing the mesher Sheetmesh is applied with 6 elements in thickness-direction and an edge length of $0.35 \mathrm{~mm}$. This mesher is chosen as the deep drawing process is a classical sheet metal forming process. In the upsetting operation a hexahedron mesh with an edge length of $0.3 \mathrm{~mm}$ is applied as upsetting belongs to the group of bulk metal forming processes. The meshing parameters are presented in Table 1.

Other than in the experimental set-up drawing die and upsetting plate are defined as active components.
Table 1: Parameters for the numerical model

\begin{tabular}{|l|l|l|}
\hline & Deep drawing & Upsetting \\
\hline Mesher length & Sheetmesh & Overlay Hex \\
\hline $\begin{array}{l}\text { Edge } \\
\text { global }\end{array}$ & $0.35 \mathrm{~mm}$ & $0.30 \mathrm{~mm}$ \\
\hline $\begin{array}{l}\text { Edge length } \\
\text { minimal }\end{array}$ & - & $0 \mathrm{~mm}$ \\
\hline $\begin{array}{l}\text { Elements over } \\
\text { thickness }\end{array}$ & 6 & - \\
\hline Edge angle & $30^{\circ}$ & $60^{\circ}$ \\
\hline Remesh & none & none \\
\hline Friction model & Tresca & Tresca \\
\hline Friction global & 0.1 & 0.1 \\
\hline $\begin{array}{l}\text { Workpiece } \\
\text { material }\end{array}$ & DC04 & DC04 \\
\hline
\end{tabular}

\subsection{Identification of the blank layout}

The part geometry requires different material volumes in different areas to enable a complete die filling. The allocation of additional material for the functional elements shall enable an increased die filling and at the same time prevent tangential material flow and increased tool loads [9]. To adapt the blank layout the material necessary to enable a complete die filling is calculated. Figure 4 shows the material requirement of the different part areas.

a) Round cup

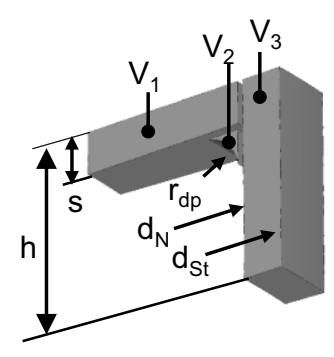

b) Open carrier

c) Gearing
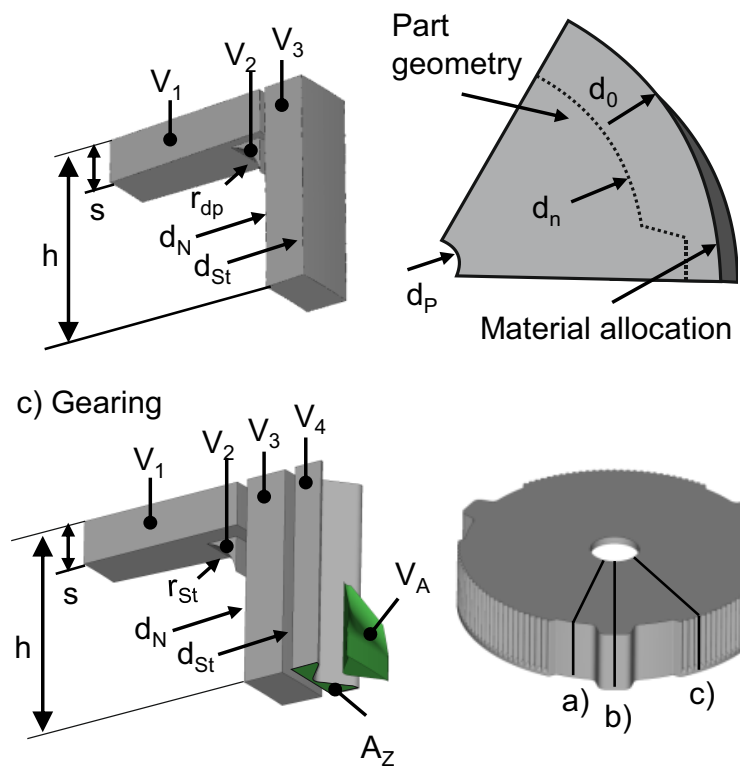

Figure 4: Determination of material requirement for round cup (a), open carrier (b) and gearing (c)

The adaption is realized by designing blanks in assumption of constant volume based on round semifinished products with an initial diameter of $\mathrm{d}_{0}=100 \mathrm{~mm}$. Therefore, the cup height $\mathrm{h}$ for a round cup without the arrangement of functional elements is determined and the required material volumes $\mathrm{V}_{1}, \mathrm{~V}_{2}$ and $\mathrm{V}_{3}$ are calculated. $\mathrm{V}_{1}$ represents the material volume in the bottom of the cup, $\mathrm{V}_{3}$ is the material required to fill the round cup. The volume $V_{2}$ is required to fill the gap at the drawing punch radius. Based on preliminary studies 
and the approach presented in [9] an adapted blank layout is identified to enhance the filling behavior for the open carrier and enable the manufacturing of a cup with homogeneous height. For the gearing the additional volumes $\mathrm{V}_{4}$ and $\mathrm{V}_{\mathrm{A}}$ are required. $\mathrm{V}_{4}$ defines the material volume for the gear teeth and the volume $\mathrm{V}_{\mathrm{A}}$ has to be considered due to the tool construction. The volumes are determined based on the cup height calculated for the round cup. Taking into account of these calculations additional material is provided by an adaption of the blank layout to allocate the material required. The material is intended to improve the die filling behavior of the open carrier and gearing during upsetting. Considering the calculations under assumption of constant volume the semi-finished product geometry shown in figure 5 is identified. The material provision for the gearing is realized by an expansion of the outer blank diameter from $100 \mathrm{~mm}$ to $110 \mathrm{~mm}$. For the open carrier material is provided by increasing the initial blank diameter by an eccentric adaption with a maximum diameter of $106 \mathrm{~mm}$. The angle of the transition between these zones is set to $\alpha=40^{\circ}$. The beginning of the transition set to the beginning of the gearing, proceeds over the first gear tooth and ends over the tooth tip of the second tooth. This ensures a complete allocation of material for the first gear tooth which lies in between the gearing and not at the transition to the round cup where less material volume is required.

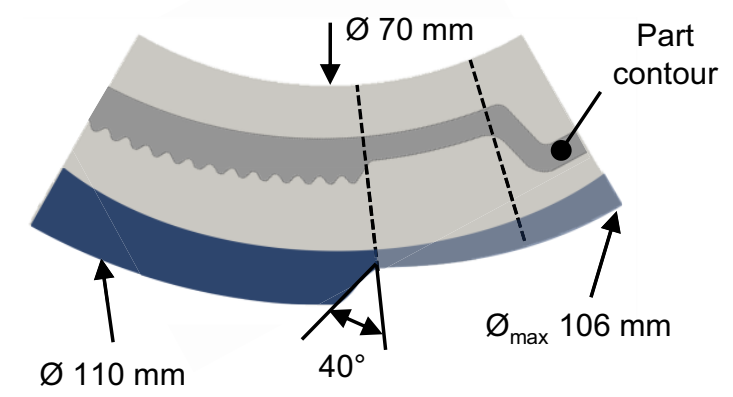

Material allocation for gearing

Material allocation open carrier

Figure 5: Initial semi-finished product geometry

An enhanced control of the induced material flow is the prerequisite for forming of near-net-shaped parts in the process combination of deep drawing and upsetting [8]. After defining the layout for the cup with increased functional integration the numerical investigation is performed to analyze the material flow in the forming process.

\section{Numerical investigations of the material flow for manufacturing of cups with various functional elements}

In the numerical investigation interactions regarding blank layout, draw-in behavior and resulting part properties after deep drawing and regarding die filling and part properties after upsetting are analyzed. To investigate the transferability of the results for cups with external gearing or open carrier and determine interactions between these functional elements an isolated consideration of the processes applied is necessary. The properties of the drawn cup have a strong influence on the material flow during upsetting and the resulting properties of the finished part. First of all, the forcedisplacement diagram is analyzed. Subsequently, the distribution of stress, tool contact and effective plastic strain are presented and discussed, to enhance the process knowledge. The numerical simulation thereby is an important tool as it enables an analysis during the forming process which is hardly possible in experimental tests.

\subsection{Draw-in behaviour and material flow during deep drawing}

The force-displacement curve for the deep drawing process is shown in figure 6 . At the beginning of the deep drawing process the forming force increases as the material is drawn in over the outer radius of the drawing die $r_{o}$. As soon as the workpiece material gets in contact with the conical drawing profile with an angle of $45^{\circ}$ the material is bent over the inner radius $r_{i}$. This results in a steeper increase of the force required due to a reduced length of the lever arm. A first maximum of the drawing force can be determined after most of the material is drawn in and afterwards the drawing force required decreases. Up to this point the curve shows the typical progression of a conventional deep drawing process. However, with ongoing process the forming of the open carrier results in a second increase of the drawing force as the material is uptight on both sides of the open carrier due to the draw-in behavior.

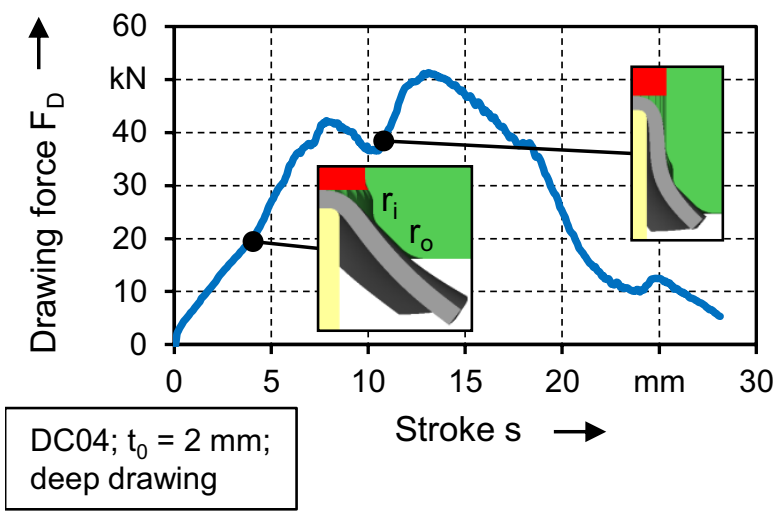

Figure 6: Force-displacement curve for deep drawing of the initial layout

The curve progression is caused by the specific stress and strain states in the functional elements and by interaction of these elements in the forming operation. In the beginning of the deep drawing process the area of the round cup is bulged between the areas with higher material volume for gearing and open carrier, as shown in figure 7. This leads to tangential compression on the outside of the cup wall and tangential tension on the inside. The forming of the open carrier leads to increased tangential tensile stresses during the ongoing drawing operation. The tangential material flow induced leads to 
increased material allocation in the outer area of the gearing in tangential direction. The angle of the transition zone amounts to $20^{\circ}$ after deep drawing from initially $40^{\circ}$ in the blank layout. The decreased transition angle leads to an increased material allocation for the first and last tooth in the gearing section, as the tangential material flow was not considered in the blank layout. The tool contact and the distribution of effective plastic strain have to be investigated during the process in the different sections, as the deep drawn cup defines the boundary conditions for the upsetting process.

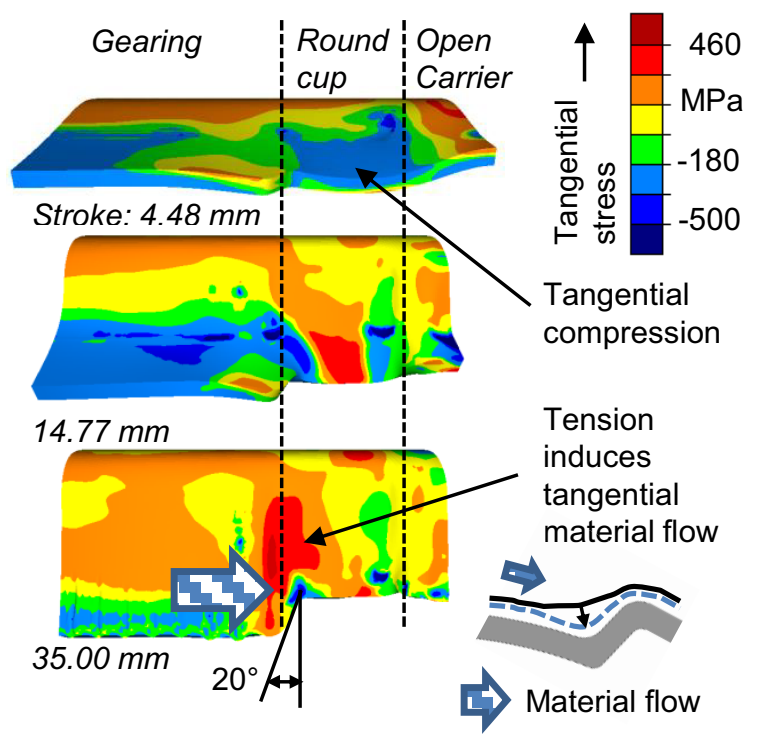

Figure 7: Draw in behavior and tangential stress states during deep drawing

The draw in behavior depends on the tool contact during the process. Table 2 shows the contact zones of tool and workpiece and the resulting development of the effective plastic strain in the part. It is shown that there is no circumferential contact between tool and workpiece. The areas of gearing and open carrier as well as the transition zones are in contact with the drawing die, whereas the area of the round cup is not due to bulging. At the beginning of the deep drawing process the highest effective plastic strain in the area of the drawing punch radius occurs at the open carrier as this zone faces radial and tangential stress and strain states. With ongoing process the drawing die moves downwards and forms the cup and open carrier. The effective plastic strain in the transition zones from round cup to the functional elements is significantly higher than in the other sections as small contact zones face high contact pressure. This leads to surface deformation due to high contact normal pressure. At the end of the deep drawing operation values of more than 1.5 are determined which are significantly higher than the values in the rest of the part. The effective plastic strain at the front of the open carrier locally surpasses 1 due to contact pressure caused by the forming of the carrier. In the outer area of the gearing in axial direction unintended material flow is induced by ironing of the cup wall. An increased wall thickness caused by tangential compression and the stiffness of the cup lead to a shaping of the gear contour into the workpiece material. Because of the tangential material flow and the smaller cavity of the round cup the values in the middle of the geared section of the part are lower than in the outer section. After deep drawing a significant difference in height between the gearing and the open carrier is detectable due to the blank geometry. The differences in height are required by the material volume necessary to fill the various functional elements in the upsetting process.

Table 2: Tool contact, distribution of effective plastic strain and stroke during deep drawing

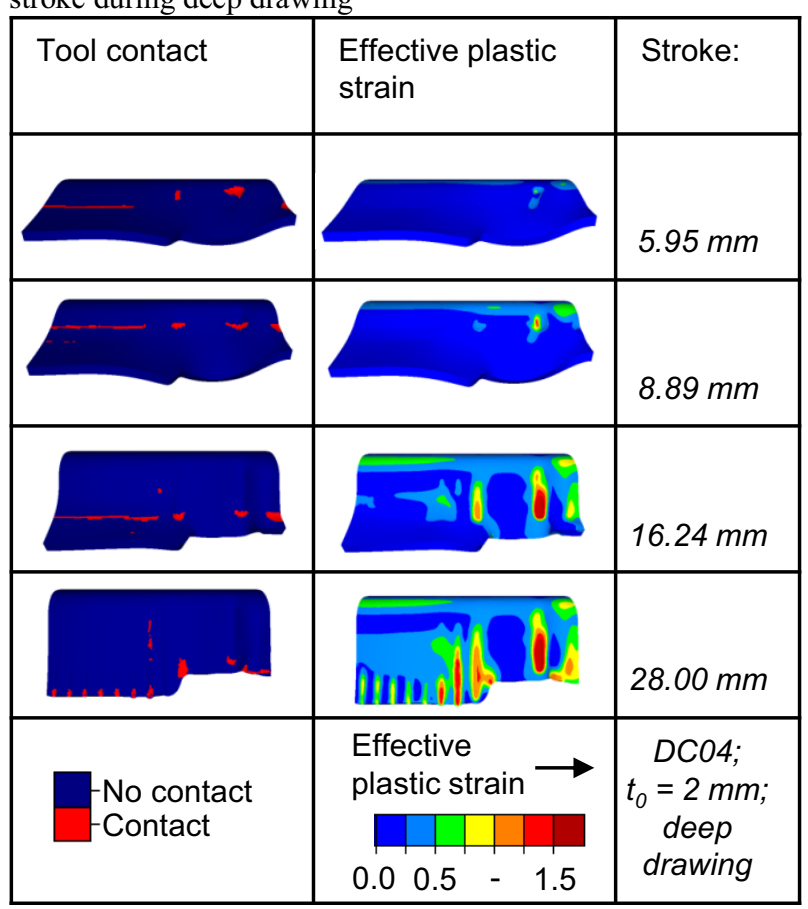

For a more detailed analysis of the part properties the distribution of effective plastic strain in the cross-section of the functional elements is investigated. The distribution of effective plastic strain in the cross section is shown in figure 8 for the different part. The crosssections show the highest deformation in the open carrier and the increased thinning in the area of the drawing punch radius due to increased tangential tensile stress states described above. For round cup and gearing the values for effective plastic strain are increased only in the area of the drawing punch due to radial stress in the outer side and compressive stress in the inner side of the bending zone. For the round cup the effective plastic strain on the inside is higher than on the outside, whereas for the gearing higher effective plastic strain on the outside occurs. This is caused by the increased material volume in the drawing gap and the ironing of the cup wall. The material in the area of the drawing punch radius is supported on the inside surface as it is in contact with the punch and ironing causes additional tensile stress on the outside surface.

The results shown for cups with combined functional elements are transferable to the results for cups with similar functional elements presented in [8] and [9]. Increased thinning of the material under radial tensile stress in the area of the drawing punch radius and thickening in the wall due to tangential compression 
occurs. Also the intensified thinning at the top of the open carrier is determined. For the cup with various functional elements the minimum sheet thickness at the drawing punch radius is $0.9 \mathrm{~mm}$ and the maximum sheet thickness in the wall is $2.2 \mathrm{~mm}$.

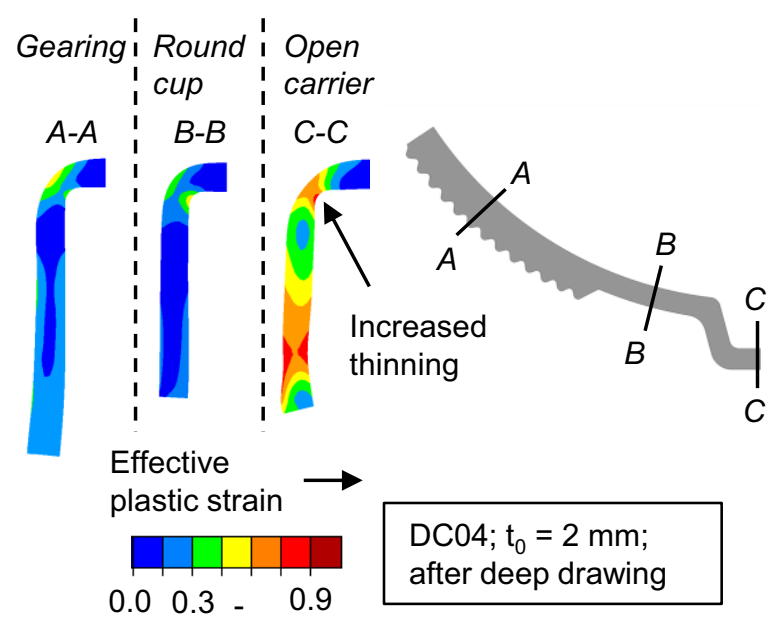

Figure 8: Effective plastic strain for the cross-sections of the functional elements after deep drawing for the initial layout

The geometry and the mechanical properties of the deep drawn cup are now transferred to the numerical model for the upsetting process. The influence of the part properties of the deep drawn cup are investigated as the cup is formed in the upsetting process.

\subsection{Captions/numbering}

To determine characteristic stages of the upsetting process the force-displacement graph is analyzed. The curve for upsetting is shown in figure 9 and rises after $\mathrm{s}=2 \mathrm{~mm}$ when the cup gets in contact with the upsetting plate. In the beginning of the upsetting operation only the area of the external gearing is upset. An increase of the gradient is determined as an even cup height is reached and open carrier and cup wall are also upset.

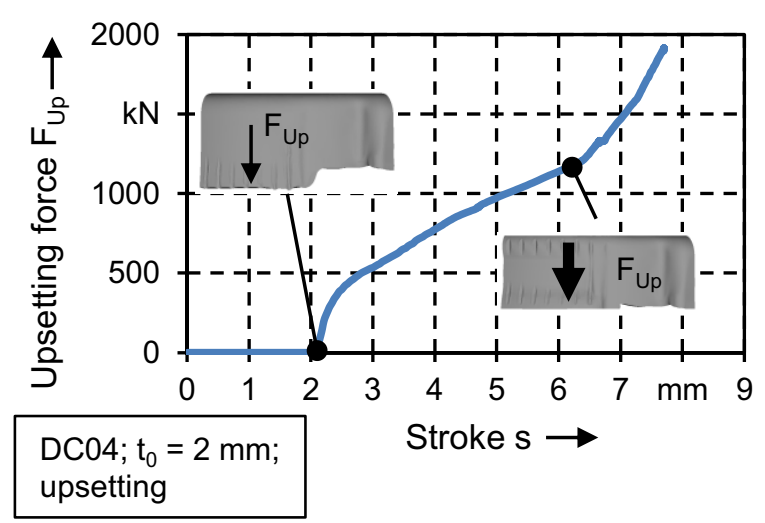

Figure 9: Force-displacement curve for upsetting of the initial layout

This is caused by the increased contact zone between tool and workpiece material which is accompanied by higher friction. Furthermore, the required upsetting force rises due to induced cold hardening in areas with high deformation. An increased die filling by applying higher upsetting forces is possible, yet not expedient as this causes high tool loads due to tangential material flow.

The distribution of elastic plastic strain is now investigated to analyze the material flow during the upsetting operation. The effective plastic strain distribution in the part as well as in the cross-sections of gearing, round cup and open carrier is analyzed. As the gearing is a thick-walled functional element it is formed by thickness increase. The material volume required is made available by radial material allocation. The resulting increased cup height after deep drawing faces axial compression and buckles. Due to the unfavorable upset ratio the cup wall buckles twice in the area of the external gearing and two grooves occur vertically to the gear teeth. This inhibits the material flow control due to inhomogeneous cold hardening and leads to worsened die filling behavior. Furthermore, the cross-sections of the part sections show high effective plastic strain at the drawing punch radius as presented in preliminary studies [8]. The decreased material thickness at the drawing punch and the high effective plastic strain are caused high deformation after deep drawing, as shown in [8]. The specific distribution of effective plastic strain for the various part sections is presented in figure 10 .

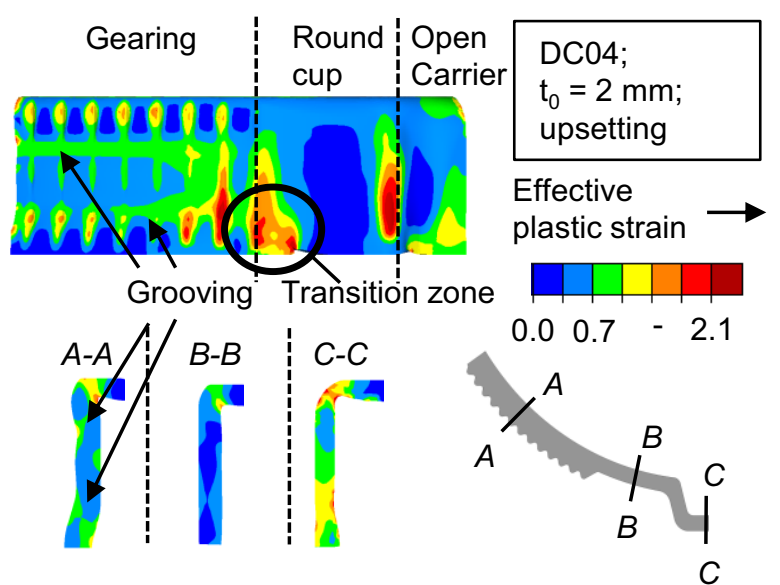

Figure 10: Effective plastic strain after upsetting of the initial layout

A zone with high effective plastic strain occurs at the transition zone from gearing to the round cup. This zone faces high tangential stress after deep drawing, as shown above. This increased tangential tensile stress and the induced lateral material flow lead to a decreased transition angle, as presented. For a closer investigation an analysis of the upsetting process at different strokes is necessary and is presented in figure 11. The transition angle amounts to $\alpha=20^{\circ}$ after deep drawing. In the upsetting operation not only the intended axial and radial material flow into the gear cavity occurs but also unintended tangential material flow in areas with minor flow resistance. As shown in the force-displacement diagram for the upsetting process only the additional material volume for the gearing is upset at the beginning of the process. The steep transition angle of $\alpha=20^{\circ}$ leads to a disadvantageous transmission of force into the area of the round cup and uncontrolled material flow. As upsetting induces lateral expansion of material the 
material allocated for the gearing becomes wider as the height is reduced. This is supported by the minor cup height in the area of the round cup as there is no material flow interference in tangential direction and causes folding of the material.
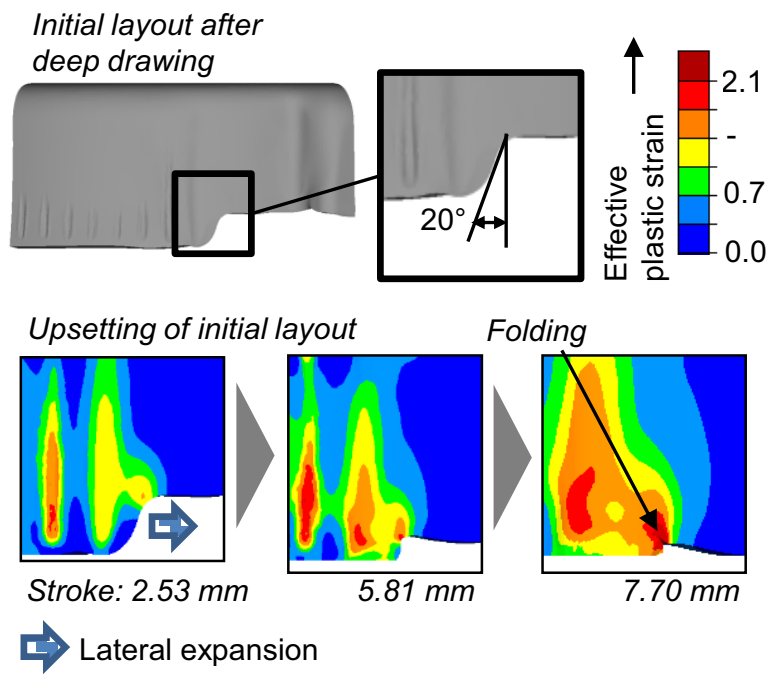

Figure 11: Folding of the initial layout in the upsetting process

Local inhomogeneous distribution of effective plastic strain in the transition zone causes problems with regard to material flow control. To prevent folding and achieve an increased homogeneity of the effective plastic strain distribution the transition angle of the blank layout is adapted.

\subsection{Adaption of the transition angle to enhance the material flow control}

The transition angle is reduced by drawing in the workpiece material in the deep drawing process and the lateral expansion during upsetting causes folding. This is considered as the layout of the semi-finished product is adapted. To prevent the folding in the transition zone the angle of the material provision for the gearing is flattened and set to $\alpha_{\mathrm{a}}=55^{\circ}$, as shown in figure 12 . The angle in the initial layout is $40^{\circ}$ and was adopted according to the tangential material flow identified.
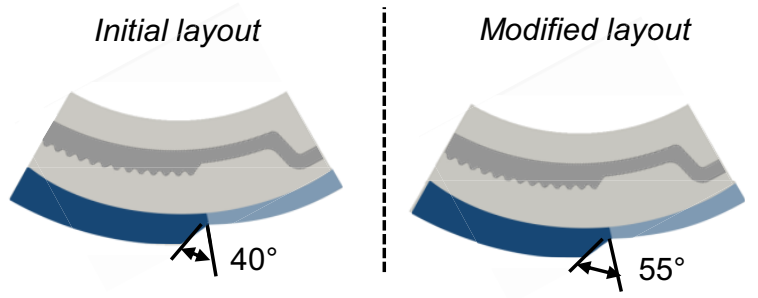

Material allocation for gearing

Material allocation open carrier

Figure 12: Adaption of blank layout

For the section between gearing and round cup a flatter transition is realized after deep drawing, as presented in figure 13. The transition angle is increased from $20^{\circ}$ to $35^{\circ}$. The decrease of $20^{\circ}$ for both cases can be explained by the tangential constant tensile stress induced by the open carrier.
Transition angle after deep drawing

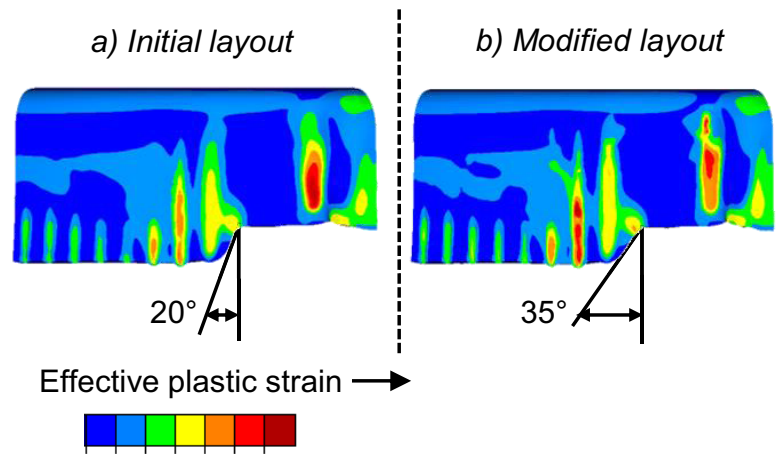

$0.00 .7-2.1$

Figure 13: Transition angle for initial (a) and modified (b) layout after deep drawing

After deep drawing the adaption of the angle only minor deviations in the distribution of effective plastic strain occur due to the draw-in behavior. However, the adaption has a positive influence on the part properties after upsetting. Due to the adaption of the transition angle the lateral extension of the material allocation for the gearing does not cause folding, as shown in figure 14 . The prevention of folding allows an increased radial material flow and thereby an improved die filling in the upsetting operation.

\section{Modified layout after upsetting}

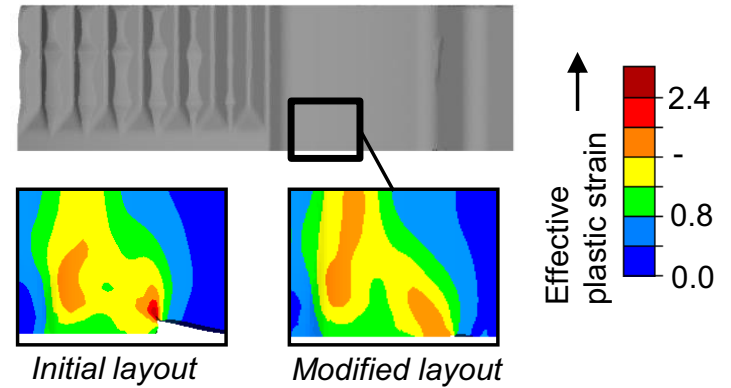

Figure 14: Effective plastic strain in the transition zone for initial (left) and modified layout (right)

\section{Experimental verification of the numerical investigation}

After numerical analysis an experimental investigation is performed to verify the numerical results as simplifications have to be made in the numerical investigation. Semi-finished products with modified layout are applied. The parts manufactured are digitalized with an optical scanner by strip projection. By measuring all 45 teeth the mean value and the standard deviation are calculated according to the arrangement of the teeth. Due to the cyclic symmetric arrangement half of the gearing is analyzed. As shown in the numerical investigation the die filling for the outer teeth of the geared section is significantly higher than for the rest of the gearing because of the interactions described above. The increased die filling of outer sides of the gearing is caused by tangential tensile stress which induces a tangential material flow and an increased material allocation at the outside of the gearing. The increase of 
the die filling in the middle of the gearing however is not significant. Figure 15 shows the mean values of the die filling for a manufactured part out of the deep drawing steel DC04 and confirms with the numerical results. The upsetting force applied is $\mathrm{F}_{\mathrm{u}}=400 \mathrm{kN}$.

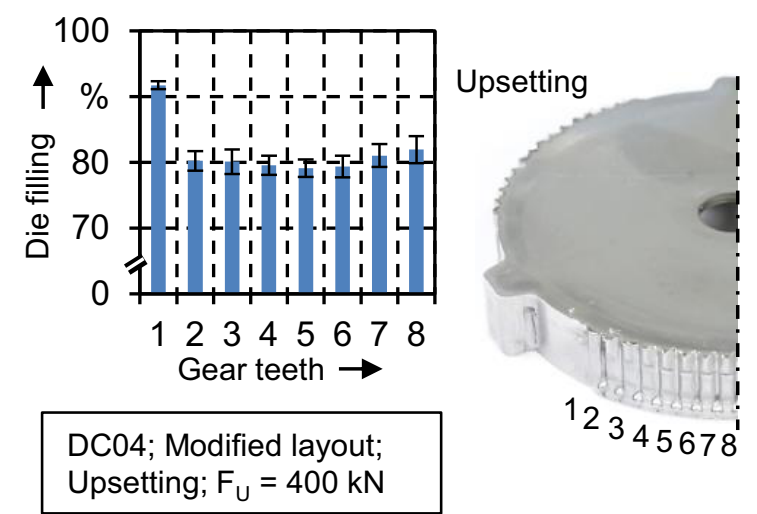

Figure 15: Die filling of gear teeth in the experimental test for DC04

The cross-sections of gearing and open carrier show the different material volume required to fill the die. The results confirm the numerical investigation as the geometrical part properties correspond to the results of the simulation. The outer contour of external gearing with circumferential grooving is shown in figure 16. a) External Gearing

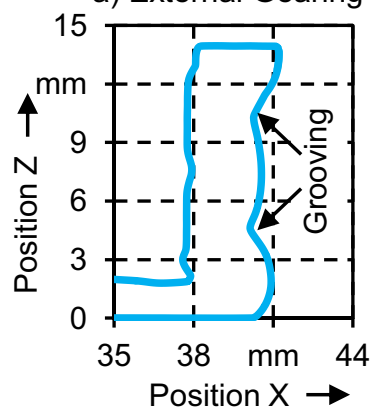

b) Open Carrier

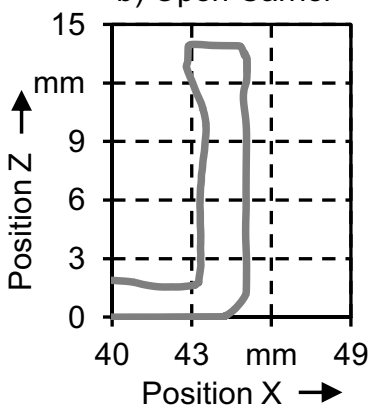

Figure 16: Cross-section of external gearing (a) and open carrier (b)

\section{Conclusion}

The functional integration of elements in a single part is shown in the numerical model as well as in the experimental test. A blank layout is constructed under assumption of constant volume. The investigation shows the interactions between different functional elements. The forming of the open carrier induces tangential stress states and tangential material flow which leads to folding in the transition zone from round cup and gearing and causes increased tool loads and insufficient die filling. An adaption of the transition angle prevents folding in this area of the part and enhances the material flow control. Also the grooving of the cup wall is analyzed. The adaption of the material allocation angle enables decreased folding in the upsetting operation. To prevent buckling of the cup wall the application of Tailored Blanks with process-adapted material thickness distribution is a promising approach. Pursuing this approach the application of Tailored Blanks has to be further investigated with regard to the material flow and the resulting part geometries.

\section{Acknowledgement}

This work was supported by the German Research Foundation (DFG) within the scope of the Transregional Collaborative Research Centre on Sheet-Bulk Metal Forming (TCRC 73, https://www.tr-73.de) in the subproject A1 (Deep Drawing).

\section{References}

1. M. Kleiner, M. Geiger, A. Klaus, CIRP Annals Manufacturing Technology, Volume 52, Issue 2, pp. 521-542 (2007)

2. J. Rowe, Advanced Materials in Automotive Engineering, Woodhead Publishing, Cambridge, UK (2012)

3. J. M. Allwood, J. M. Cullen, Sustainable Materials With Both Eyes Open, UIT Cambridge Ltd., Cambridge, UK (2011)

4. http://www.umformtechnikmagazin.de/umformtechn ik-fachartikel/massivumformung-vonblech_19204_de/ (accessed January $\left.26^{\text {th }} 2016\right)$

5. T. Takami, Procedia Engineering 81, pp. 5-17 (2014)

6. M. Merklein, J.M. Allwood, B.A. Behrens, A. Brosius, H. Hagenah, K. Kuzmann, K. Mori, A.E. Tekkaya, A. Weckenmann, CIRP Annals Manufacturing Technology 61-2, pp. 725-745 (2012)

7. K. Mori, Steel Research International - Special Edition $14^{\text {th }}$ International Conference Metal Forming, pp. 17-24 (2012)

8. T. Schneider, U. Vierzigmann, M. Merklein, AIP Conference Proceedings, Volume 1567, Issue 1, pp.930-933 (2013)

9. M. Merklein, M. Löffler, T. Schneider, CIRP Annals - Manufacturing Technology, 64-1, pp. 245-248 (2015) 